\section{Lipodistrofias primarias: presentación clínica y diagnóstico}

\author{
VÍCTOR CORTÉS ${ }^{1}$, JOSÉ LUIS SANTOS
}

\section{Clinical presentation and treatment of primary lipodystrophies}

Lipodystrophies are a heterogeneous group of syndromes defined by a severe reduction of the adipose tissue. These can be congenital or acquired. Anatomically, they can be partial or generalized. The etiology of several lipodystrophies is well known. However, the cause of many others remains unknown. The commonest lipodystrophy worldwide is secondary to highly active anti-retroviral therapy in HIV-infected patients. By contrast, primary lipodystrophies (those not associated to any known disease or condition) are much less common and represent a diagnostic challenge. The major complications of lipodystrophies are metabolic, often resulting in severe insulin resistance, diabetes and dyslipidemia. No cure is available for lipodystrophies but the supplementation with recombinant leptin potently controls the metabolic abnormalities when there is a leptin deficiency. Herein, we review the clinical presentation, diagnostic process and therapeutic principles of the main primary lipodystrophy syndromes.

(Rev Med Chile 2019; 147: 1449-1457)

Key words: Adipose Tissue; Diabetes Mellitus; Insulin Resistance; Leptin; Lipodystrophy.

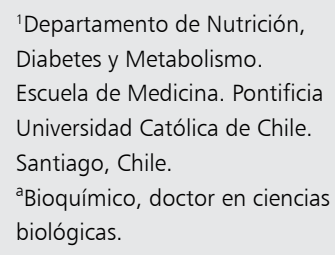

Los autores declaran no tener conflictos de interés.

V.A.C y J.L.S. reciben

financimiento Fondecyt, códigos 1181214 y 1150416 , respectivamente.

Recibido el 5 de febrero de 2019, aceptado el 28 de agosto de 2019.

Correspondencia a:

Víctor Cortés

Departamento de Nutrición,

Diabetes y Metabolismo

Escuela de Medicina

Pontificia Universidad Católica de

Chile. Santiago, Chile.

vcortesm@uc.cl

\section{I} as lipodistrofias son síndromes heterogéneos, definidos por reducción del tejido $\checkmark$ adiposo. En casos de lipodistrofia generalizada, esta reducción puede determinar una carencia virtualmente total de adiposidad corporal. Por el contrario, en casos de lipodistrofia parcial, la masa adiposa total puede estar disminuida o bien ser normal o incluso estar aumentada, como consecuencia de redistribución del tejido adiposo remanente y lipohipertrofia segmentaria.

Los pacientes lipodistróficos desarrollan complicaciones metabólicas secundarias a resistencia insulínica, que pueden ser graves y de difícil manejo.

Desde una perspectiva biológica, las lipodistrofias pueden ser entendidas como el resultado de procesos anormales en el desarrollo, crecimiento o función del tejido adiposo. Así, su estudio ha permitido incrementar el conocimiento de la biología normal y patológica de este tejido ${ }^{1-4}$, lo cual es relevante en el contexto epidémico actual de obesidad. En efecto, la paradoja de que tanto el exceso de tejido adiposo (obesidad) como su deficiencia (lipodistrofia) determinan idénticas anormalidades metabólicas, indica que los mecanismos fisiopatológicos de resistencia insulínica son solo parcialmente comprendidos en la actualidad ${ }^{5}$.

\section{Clasificación y etiología de las lipodistrofias}

Las lipodistrofias primarias, es decir, aquellas que no son consecuencia de un proceso patológico de base, son enfermedades infrecuentes ${ }^{6}$. En la literatura existen aproximadamente 1.000 pacientes reportados con lipodistrofias de causa genética, estimándose una prevalencia global en 1:10.000.000 en la población general ${ }^{7}$.

Anatómicamente, las lipodistrofias pueden ser generalizadas o parciales. También existen formas "localizadas", que corresponden a atrofia del tejido 
adiposo subcutáneo en áreas muy restringidas. Son causadas por noxas repetidas, como inyecciones, presión prolongada o traumatismos, y no tienen consecuencias metabólicas.

Aun cuando la etiología de muchas lipodistrofias es desconocida, han sido clasificadas como "congénitas" o "adquiridas".

\section{Manifestaciones clínicas de las lipodistrofias primarias}

Las anormalidades metabólicas de las lipodistrofias son hiperinsulinemia, hiperglicemia, hipertrigliceridemia o dislipidemia mixta, esteatosis hepática y ovario poliquístico. Además, dependiendo de la magnitud de la reducción de la masa adiposa total y de la etiología específica, pueden observarse niveles reducidos o ausencia de hormonas de origen adipocitario (denominadas adipoquinas), claves para la regulación del metabolismo energético, lipídico y glucídico. La deficiencia de estas hormonas, entre las que destaca leptina, juega un rol central en la fisiopatología de la resistencia insulínica e hiperfagia de los síndromes lipodistróficos ${ }^{8}$.

Adicionalmente, pueden presentarse miopatías (cardiacas y esqueléticas) ${ }^{9}$, nefropatías ${ }^{10}$ y trastornos neurológicos que, según el conocimiento actual, no serían resultado directo de las patologías metabólicas basales, sino que de otros defectos derivados de las acciones biológicas de los genes afectados.

\section{A. Lipodistrofias genéticas}

Estas lipodistrofias pueden ser generalizadas o parciales. Las lipodistrofias congénitas generalizadas (LCG) se caracterizan por reducción global del tejido adiposo corporal. Las lipodistrofias familiares parciales (LFP) consisten en lipoatrofia marcada de las extremidades, frecuentemente asociada a lipohipertrofia segmentaria del tejido adiposo del tronco, cuello y cabeza. Existe también un espectro de condiciones genéticas de presentación compleja y que, entre otras manifestaciones, presentan lipodistrofia. Entre ellas destacan varios síndromes progeroides y síndromes autoinflamatorios tales como dermatosis neutrofílica atípica crónica con lipodistrofia y elevación de temperatura (CANDLE) y contracturas articulares con atrofia muscular, anemia microcítica y lipodistrofia inducida por paniculitis (JMP) (Tabla 1).

\section{1) Lipodistrofias congénitas generalizadas (sindrome de Berardinelli-Seip)}

Consisten en reducción del tejido adiposo subcutáneo y visceral de todo el organismo y son las formas más graves de lipodistrofia (Figura 1). Fueron descritas por primera vez en 1954, en Brasil, por W. Berardinelli ${ }^{11}$ y en Noruega por M. Seip, en $1959^{12}$. Tienen transmisión autosómica recesiva $y$, a la fecha, se han reportado aproximadamente 300 casos en la literatura. Existen cuatro subgrupos, dependiendo de los genes afectados ${ }^{7}$. Las LCG-1 y -2 son causadas por mutaciones en los genes AGPAT2 y BSCL2, respectivamente, y corresponden a $95 \%$ de los casos conocidos. Las LCG-3 y -4 son causadas por mutaciones en CAV1 y PTRF, respectivamente, y presentan características fenotípicas adicionales (Tabla 1).

Los signos cardinales de LCG son: 1) lipoatrofia grave evidente desde el nacimiento o los primeros días de vida; 2) acantosis nigricans de aparición en la infancia; 3) hiperfagia asociada a apetito muy intenso; 4) hiperlipidemia, usualmente mixta, pero predominantemente hipertriglicéridemica; 5) hiperinsulinemia; 6) diabetes mellitus, habitualmente desde la adolescencia y rara vez desde la infancia; 7) reducción marcada de adipoquinas circulantes, con leptina y adiponectina frecuentemente indetectables.

Otros elementos de manifestación menos consistente incluyen crecimiento longitudinal acelerado, hirsutismo, pseudoacromegalia y clitorimegalia.

Las mujeres con LCG presentan frecuentemente hiperandrogenismo, oligoamenorrea, ovario poliquístico e infertilidad. Hay muy pocos casos reportados de embarazos exitosos, siendo uno de ellos una paciente chilena diagnosticada en nuestro centro ${ }^{13,14}$. Los hombres afectados tienen fertilidad preservada ${ }^{7}$.

Los pacientes con LCG frecuentemente desarrollan proteinuria ${ }^{15}$. Sorprendentemente, la nefropatía diabética es un hallazgo poco frecuente en estos pacientes ${ }^{10}$, siendo más comunes la glomeruloesclerosis focal y segmentaria y la glomerulonefritis membranoproliferativa ${ }^{13,16,17}$. Las causas de la enfermedad renal en LCG son desconocidas, pero, tanto la proteinuria como los defectos de filtración glomerular son aminorados por la suplementación con leptina ${ }^{15,18}$. No hay claridad de si estos efectos beneficiosos dependen de la acción directa de leptina sobre los distintos tipos celula- 
Tabla 1. Comparación de las causas y fenotipo de las principales lipodistrofias primarias

\begin{tabular}{|ll}
\hline Tipo & Causa \\
$\begin{array}{l}\text { Lipodistrofia } \\
\text { congénita } \\
\text { generalizada } \\
\text { (LCG, síndrome de } \\
\text { Berardinelli-Seip) }\end{array}$ & $\begin{array}{l}\text { Mutaciones homocigotas en genes AGPAT2 } \\
\text { (LCG1), BSCL2 (LCG2), CAV1 (LCG3), PTRF (LCG4) }\end{array}$ \\
& \\
& \\
$\begin{array}{l}\text { Lipodistrofia } \\
\text { adquirida } \\
\text { generalizada } \\
\text { (LAG, síndrome de } \\
\text { Lawrence) }\end{array}$ & $\begin{array}{l}\text { Causa desconocida, ha sido asociada a autoin- } \\
\text { munidad en otros sistemas pero existen casos sin }\end{array}$ \\
$\begin{array}{ll}\text { Lipodistrofia } \\
\text { familiar parcial }\end{array}$ & $\begin{array}{l}\text { Tipo 1 (síndrome de Kobberling): causa desco- } \\
\text { nocida. Historia familiar de lipodistrofia poco }\end{array}$ \\
& $\begin{array}{l}\text { consistente, con casos esporádicos reportados } \\
\text { Tipo 2 (variante Dunnigan), mutaciones heteroci- } \\
\text { gotas en gen LMNA, con transmisión autosómica } \\
\text { dominante }\end{array}$ \\
& $\begin{array}{l}\text { Tipo 3: mutaciones heterocigotas en el gen } \\
\text { PPARG, transmisión autosómica dominante }\end{array}$ \\
& Tipo 4: mutaciones heterocigotas en el gen \\
& PLIN1, transmisión autosómica dominante
\end{tabular}

Tipo 5: mutaciones homocigotas en el gen CIDEC, transmisión autosómica recesiva

Tipo 6: mutaciones homocigotas en el gen LIPE, transmisión autosómica recesiva

\section{Fenotipo}

Reducción marcada de la adiposidad corporal total desde el nacimiento y asociada a resistencia insulínica grave. Adicionalmente puede haber: 1) deterioro cognitivo (LCG2), 2) baja estatura, hipocalcemia, hipomagnesemia, resistencia a vitamina D (LCG3), 3) miopatía esquelética y cardiaca, arritmias cardiacas, estenosis pilórica (LCG4)

Reducción marcada de la adiposidad corporal asociada a resistencia insulínica grave en personas con adiposidad previamente normal. Suele iniciarse en la pubertad en conjunto con paniculitis y otras manifestaciones de autoinmunidad

Lipoatrofia de extremidades asociada a lipohipertrofia troncal

Lipoatrofia de extremidades con lipohipertrofia de cuello y cara. Adiposidad troncal puede estar aumentada, al igual que la vulvar en mujeres

Lipoatrofia marcada de extremidades, más intensa en regiones distales

Lipoatrofia progresiva de extremidades, que afecta mayormente las extremidades inferiores y glúteos, y asociada a infiltración macrofágica y fibrosis del tejido adiposo

Lipoatrofia de extremidades, más intensa en extremidades inferiores. Anormalidades metabólicas menores en la infancia pero que progresan a hipertrigliceridemcia grave en edades mayores Lipoatrofia marcada de extremidades inferiores con lipohipertrofia de cara, cuello, supraclavicular, axilar, abdomen y vulva. Miopatía progresiva de aparición en la adultez. Resistencia insulínica variable

Lipodistrofia Causa desconocida pero presumiblemente autoinadquirida parcial (síndrome de mune dada su asociación en algunos casos con enfermedades autoinmunes clásicas como LES y Barraquer Simons) dermato-polimiosistis de otros sistemas

Lipoatrofia marcada y progresiva de cara, cuello, extremidades superios y tronco. La adiposidad de las extremidades inferiores y regiones glúteas está típicamente preservada o incluso aumentada en algunos casos. Las complicaciones metabólicas son menores pero es frecuente su asociación con glomerulopatía membranoproliferativa

Lipodistrofia asociada a síndromes progeroides

Otras lipodistrofias de causa genética
Diversas mutaciones en los genes LMNA, ZMPSTE24, POLD1, WRN, FBN1, BANF1, KCNJ6, SPRTN

Variantes patológicas en los genes PSMB8, PIK3R1 y MFN2 en estado homocigoto o heterocigoto compuesto, determinan los síndromes CANDLE (chronic atypical neutrophilic dermatosis with lipodystrophy and elevated temperature), SHORT (short stature, joint hyperextensibility, ocular depression, Rieger anomaly, and teething delay) y JMP (joint contractures, muscle atrophy, microcytic anemia, and panniculitis-induced lipodystrophy), respectivamente
Amplia variedad de dismorfismos craneofaciales, esqueléticos y cutáneos, asociados a miopatías y defectos neurológicos centrales y periféricos

Variadas anormalidades musculoesqueléticas, oculares, dentales, cutáneas e inmunológicas

Los genes mencionados en esta tabla codifican los siguientes productos: 1-acyl-sn-glycerol-3-phosphate acyltransferase (AGPAT2), Seipin (BSCL2), Caveolin 1 (CAV1), Cavin 1 (PTRF), Lamin A/C (LMNA), Peroxisome proliferator-activated receptor gamma (PPARG), Perilipina 1 (PLIN1), Cell death inducing DFFA like effector C (CIDEC), Lipase E/lipasa homonosensible (LIPE), Zinc Metallopeptidase STE24 (ZMPSTE24), DNA Polymerase Delta 1, Catalytic Subunit (POLD1), Werner Syndrome RecQ Like Helicase (WRN), Fibrillin 1 (FBN1), Barrier to autointegration factor 1 (BANF1), Potassium voltage-gated channel subfamily J member 6 (KCNJ6), SprT-like N-terminal domain (SPRTN), Proteasome subunit beta 8 (PSMB8), Phosphoinositide-3-kinase regulatory subunit 1 (PIK3R1), Mitofusin 2 (MFN2). 


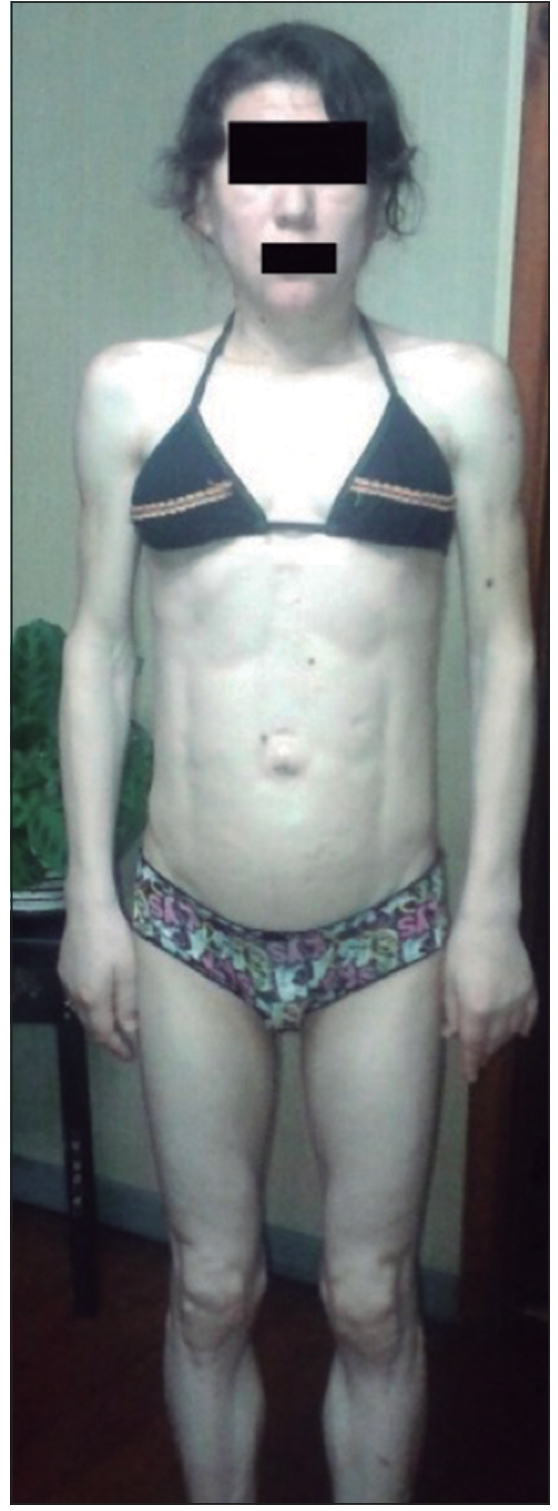

Figura 1. Paciente con lipodistrofia congénita generalizada (LCG) causada por mutaciones en el gen AGPAT2. La paciente fue diagnósticada formalmente a los 29 años de edad, pero a los 13 años de edad debutó con diabetes mellitus, dislipidemia mixta grave (colesterol total $=960 \mathrm{mg} / \mathrm{dl}$, triglicéridos $=4.355 \mathrm{mg} / \mathrm{dl}$ ) e hígado graso. Al momento del diagnóstico de LCG, la paciente presentaba retinopatía proliferativa grave en ambos ojos y proteinuria de 2,8 g/día. Como es evidente al examen físico, la paciente presenta una marcada reducción de la adiposidad subcutánea, que determina una demarcación notoria de las masas musculares. Además, son apreciables algunos razgos acromegaloides (prognatismo y agrandamiento de manos y pies) y una hernia umbilical pequeña. Detalles del estudio genético y fenotipíco de esta paciente y su hermana pueden encontrarse en la referencia 43. res del riñón, o si son consecuencia de la mejoría de parámetros hemodinámicos y metabólicos sistémicos. Tampoco se conoce si el tratamiento con leptina revierte el daño estructural del riñón o si sus efectos son mayormente funcionales. Sin embargo, considerando que leptina reduce en $83 \%$ la albuminuria y en $56 \%$ la proteinuria en los primeros 24 meses de tratamiento ${ }^{18}$, es esperable que este tratamiento provea protección renal a los pacientes con LCG, tal como lo sugiere un metaanálisis reciente ${ }^{19}$.

Distintas manifestaciones de enfermedad cardiovascular han sido reportadas en pacientes con LCG. En un análisis de 33 casos publicados, Khalife y cols. encontraron que $97 \%$ de ellos presentaba cardiomiopatía hipertrófica (70\% simétrica y 30\% asimétrica) y que más de $50 \%$ tenía disfunción diastólica ${ }^{20}$. Por el contrario, pocos pacientes de esta serie tenían hipertensión arterial o falla cardiaca congestiva. Notablemente, pese a que estos pacientes frecuentemente evolucionan con dislipidemia y diabetes mellitus grave, ninguno de estos casos tuvo evidencia angiográfica de enfermedad coronaria aterosclerótica ${ }^{20}$. Concordantemente, en una paciente de 47 años de edad con LCG y diabetes mellitus grave, que fue sometida a trasplante renal, no se encontró evidencia macroscópica de aterosclerosis ilíaca durante la cirugía ${ }^{13}$. No obstante, no hay estudios que hayan objetivado esta aparente resistencia a la aterosclerosis en LCG.

\section{2) Lipodistrofias familiares parciales (LDFP)}

Fueron descritas por primera vez en 1885 por Wier-Mitchell ${ }^{21}$ y se caracterizan por lipoatrofia regional, a veces asociada a lipohipertrofia en áreas corporales. Estas enfermedades pueden iniciarse en la infancia, pubertad o adultez temprana $y$, al igual que la LCG, se asocian a patología metabólica. Las complicaciones cardiacas son frecuentes ${ }^{22}$. Las mujeres afectadas tienen, en general, fertilidad preservada, aunque aproximadamente $25 \%$ evoluciona con irregularidades menstruales e hirsutis$\mathrm{mo}^{23}$. Actualmente se reconocen 6 subtipos, según su etiología genética específica.

LDFP-1. Es denominada síndrome de Kobberling ${ }^{24}$ y se caracteriza por deficiencia marcada de tejido adiposo subcutáneo exclusivamente en extremidades y glúteos. La adiposidad está preservada o incluso puede estar aumentada a nivel troncal. Estos pacientes desarrollan diabetes mellitus e hipertrigliceridemia con frecuencia ${ }^{25}$. 
Su patrón hereditario no está definido y, pese a que existe agregación familar, se han descrito casos de aparición esporádica. Su etiología es desconocida y es más común en mujeres ${ }^{25}$.

LDFP-2. Es conocida como síndrome o variante de Dunnigan ${ }^{26}$ y su frecuencia estimada es de 1 en 15 millones en la población general ${ }^{27}$. Fenotípicamente, estos pacientes tienen reducción marcada de tejido adiposo subcutáneo de las extremidades, y se inicia característicamente en la pubertad. Habitualmente existe lipohipertrofia en el cuello, fosas supraclaviculares y cara, confiriendo un aspecto cushingoide. En mujeres puede haber lipohipertrofia vulvar. El tejido adiposo intraabdominal, intratorácico, de la médula ósea, palmar, plantar y orbital está conservado ${ }^{28}$. Diabetes mellitus e hipertrigliceridemia son frecuentes, pero el síndrome de ovario poliquístico no es un hallazgo común ${ }^{29}$.

Estudios de asociación han identificado diversas mutaciones en el gen $L M N A$, codificante para las proteínas lámina $\mathrm{A} / \mathrm{C}$, como la etiología genética probable de esta enfermedad ${ }^{30,31}$. Estas proteínas son esenciales para la estructura y función de los núcleos celulares y mutaciones en otras regiones de LMNA determinan variados síndromes progeroides y miopatías progresivas.

LDFP-3 y -4. Son causadas por mutaciones heterocigotas en los genes $P P A R G^{32}$ y $P L I N 1^{33}$, respectivamente, $\mathrm{y}$ tienen transmisión autosómica dominante.

LDFP-5 y -6. Fueron descritas en pacientes con mutaciones homocigotas en los genes $C^{2} E^{34}$ y $L I P E^{35}$, respectivamente, y tienen transmisión recesiva. Hay muy pocos casos descritos con estas lipodistrofias y sus características fenotípicas no están bien definidas. Sin embargo, pese a que la lipoatrofia parece ser menos grave que en la LDFP2, presentan frecuentemente diabetes mellitus y dislipidemias.

Se han reportado casos de lipodistrofia en cara y parte superior del cuerpo, asociada a micrognatia, catarata congénita e hipertrigliceridemia grave. En estos pacientes hay mutaciones con cambio del marco de lectura del gen $C A V 1^{36}$, codificante para una proteína clave para la formación de caveolas en la membrana plasmática.

\section{B. Lipodistrofias adquiridas}

Anatómicamente, pueden ser generalizadas o parciales y también son más frecuentes en mujeres. La lipodistrofia adquirida generalizada (LAG) ha sido descrita en aproximadamente 100 pacientes, la mayoría de ascendencia caucásica ${ }^{37}$. La lipodistrofia adquirida parcial (LAP) ha sido reportada en 250 casos de variados orígenes étnicos ${ }^{38}$.

\section{1) Lipodistrofia adquirida generalizada (LAG) - sindrome de Lawrence}

Estos pacientes nacen con cantidad y distribución normal de su tejido adiposo, que desaparece típicamente durante la infancia tardía o pubertad. La degeneración del tejido adiposo ocurre a lo largo de meses o años, aun cuando puede evolucionar agresivamente en el curso de semanas, determinando un síndrome de lipoatrofia grave. Esta enfermedad afecta el tejido subcutáneo de grandes áreas corporales, empezando por la cara y las extremidades, y puede incluso afectar palmas y plantas. El nivel de pérdida del tejido adiposo intraabdominal es variable, lo mismo que el de la médula ósea y órbitas ${ }^{37}$.

La etiología y los mecanismos fisiopatológicos de esta enfermedad son desconocidos. En una serie de 79 pacientes se encontró que $25 \%$ de ellos había tenido paniculitis previa al desarrollo de la lipodistrofia. En otro $25 \%$ se encontró coexistencia de enfermedades autoinmunes, principalmente dermatomiositis y hepatitis autoinmune, mientras que en el 50\% restante no se encontraron asociaciones patológicas significativas ${ }^{37}$. En algunos casos con hepatitis crónica se han reportado bajos niveles séricos de complemento $\mathrm{C} 4^{39}$, sugerentes de activación de la vía clásica del complemento.

$\mathrm{Al}$ igual que en la LCG, estos enfermos desarrollan resistencia insulínica, diabetes mellitus, esteatosis hepática y dislipidemia, por lo general grave.

\section{2) Lipodistrofia adquirida parcial (LDAP) - sindrome de Barraquer-Simons}

Esta enfermedad se caracteriza por pérdida progresiva y simétrica del tejido adiposo subcutáneo en el curso de meses o años. Habitualmente comienza en la pubertad y su progresión es cefalocaudal, típicamente preservando la adiposidad de las extremidades inferiores. Incluso, estos pacientes pueden presentar lipohipertrofia de las regiones abdominal baja, glútea, muslos y piernas. Las alteraciones metabólicas asociadas a LDAP son de menor intensidad y su principal comorbilidad descrita es la enfermedad renal crónica, principalmente glomerulonefritis membranoproliferativa ${ }^{40,41}$. 
Aunque las causas de esta enfermedad se desconocen, evidencia circunstancial sugiere que procesos autoinmunes podrían estar involucrados. Así, $80 \%$ de los casos tiene niveles séricos reducidos de complemento C3 y son positivos para el autoanticuerpo "factor nefrítico C3"38.

\section{Diagnóstico y tratamiento de las lipodistrofias}

La cantidad y de distribución del tejido adiposo pueden ser estimadas en el examen físico y sus anormalidades son evidentes en casos de lipodistrofia generalizada. Por el contrario, las lipodistrofias parciales pueden ser fácilmente confundidas con obesidad abdominal y síndrome metabólico corriente, especialmente en hombres.

De hecho, es posible que las lipodistrofias parciales sean entidades subdiagnosticadas. Apoyando esta hipótesis, un estudio multicéntrico de 5.000 pacientes diabéticos ambulatorios encontró que, entre aquellos con índice de masa corporal $<27 \mathrm{~m}^{2} / \mathrm{kg}$ y altos requerimientos de insulina (> 100 U/día), 5 tenían LDFP no previamente identificada ${ }^{42}$. Dutour y cols. mostraron que $10 \mathrm{de}$ 87 pacientes con síndrome metabólico reclutados en un servicio ambulatorio de medicina, tenían anormalidades morfológicas en los núcleos celulares y distribución subcelular anormal de lámina A/C. De estos, tres pacientes portaban mutaciones heterocigotas en LMNA y ZMPSTE24, que no habían sido previamente descritas en la literatura. Notablemente, estos 10 pacientes no eran clínicamente distintos de aquellos con síndrome metabólico común, excepto en que eran más jóvenes y tenían mayores niveles de triglicéridos plasmáticos y hepáticos ${ }^{43}$.

En pacientes con lipodistrofia parcial, el motivo de consulta suele ser alguna complicación metabólica, particularmente diabetes mellitus, y no las alteraciones anatómicas del tejido adiposo, posiblemente por el nulo conocimiento de estos síndromes en la población general.

En todo paciente lipodistrófico se debe cuantificar el estatus glicémico y la sensibilidad insulínica, así como los lípidos plasmáticos. El estudio de las funciones hepática y renal es relevante, dada la alta prevalencia de estatohepatitis no alcohólica y nefropatía proteinúrica. Los niveles circulantes de complemento $\mathrm{C} 3$ y $\mathrm{C} 4$, y de factor nefrítico
C3, deben ser determinados en casos de LDAP y LAG. La imagenología del esqueleto puede revelar lesiones líticas focales en casos de LCG. La función cardiaca debe ser examinada con electrocardiograma, Holter de ritmo o ecocardiografía en casos en que se sospeche cardiopatía. Los niveles circulantes de leptina permiten estimar la magnitud de la depleción adiposa y estimar la posible respuesta metabólica a la terapia de reemplazo con leptina recombinante.

La masa total y regional de tejido adiposo puede cuantificarse por absorbanciometría de rayos $\mathrm{X}$ de energía dual (DEXA) o resonancia magnética nuclear. En caso de definirse la necesidad de diagnóstico genético, primero deben secuenciarse los genes conocidamente asociados a lipodistrofias específicas, orientándose según la presentación clínica del síndrome lipodistrófico específico. De no resultar informativo, el estudio genético puede expandirse a la secuenciación del exoma o genoma completo. En ambos casos, el análisis genómico comparativo con los progenitores y hermanos no afectados incrementa la probabilidad de definir variantes genéticas patogénicas. Recientemente, en nuestro laboratorio hemos caracterizado genética y metabólicamente a cuatro pacientes chilenas con lipodistrofia: dos hermanas con LCG secundaria a mutaciones hereditarias del gen $A G P A T 2^{44}$, una paciente con lipodistrofia parcial de la variante Dunnigan, secundaria a mutaciones de novo en el gen $L M N A$ y una paciente con LAG, con múltiples manifestaciones de autoinmunidad.

El diagnóstico diferencial de las lipodistrofias incluye síndromes consuntivos (caquexia) de etiologías variadas, anorexia nervosa, síndrome de Cushing, obesidad troncal y síndromes infrecuentes, como la lipomatosis múltiple o síndromes progeroides atípicos.

En las lipodistrofias generalizadas, el manejo convencional con intervenciones dietéticas, actividad física y farmacoterapia es habitualmente insuficiente para el control metabólico. Debe considerarse que la depleción de tejido adiposo e hipoleptinemia resultante determinan apetito intenso e hiperfagia ${ }^{45}$, dificultado el manejo nutricional. La alimentación de pacientes con LCG debe ser normocalórica y con aporte balanceado de macro y micronutrientes. En casos de hipertrigliceridemia grave es necesaria la restricción de grasas alimentarias a menos de $15 \%$ del aporte calórico total. Por otro lado, en casos de diabetes 
mellitus grave, la restricción de carbohidratos totales también es necesaria. La actividad física debe ser suficiente para promover insulinosensibilización, sin embargo, previo a su inicio es necesaria una evaluación cardiovascular detallada, dada la frecuente asociación con cardiomiopatía. La farmacoterapia para el manejo de la resistencia insulínica, diabetes mellitus y dislipidemias, es análoga a la de pacientes sin lipodistrofia, no existiendo evidencia que permita recomendar un esquema por sobre otro.

El manejo de las complicaciones metabólicas en lipodistrofias parciales no difiere del de individuos con formas corrientes de resistencia insulínica, diabetes mellitus y dislipidemias. Incluso, se han reportado resultados de cirugía bariátrica (bypass gástrico con $\mathrm{Y}$ de Roux) en pacientes con LDFP1 y LDFP2 con buenos resultados metabó$\operatorname{licos}^{46-48}$.

Metreleptina es una forma de leptina humana recombinante de vida media larga y es la única droga aprobada para el manejo de las complicaciones metabólicas de la lipodistrofia. Desde su primer uso en lipodistrofia, en el año $2002^{49}$, se ha acumulado evidencia clínica y experimental que avala su gran efectividad terapéutica en casos de lipodistrofia generalizada, así como su buen perfil de seguridad ${ }^{8}$. Su eficacia en pacientes con lipodistrofia parcial es, en general, menor e inconsistente, por lo que solo debiera considerase en pacientes con hipoleptinemia. Los principales efectos secundarios de la metreleptina son anorexia dosis-dependiente, que puede determinar enflaquecimiento excesivo y desarrollo de anticuerpos neutralizantes con un consiguiente decremento de la eficacia de esta droga ${ }^{50}$. Adicionalmente, se han reportado casos de linfoma posteriores al inicio de metreleptina, sin embargo, dado el escaso número de pacientes tratados y la baja incidencia de esta complicación no ha sido posible establecer una relación de posible causalidad ${ }^{8}$.

\section{Lipodistrofia y diabetes mellitus tipo 2}

Se ha descrito que algunos pacientes con diabetes mellitus tipo 2 presentan un síndrome caracterizado por lipoatrofia simétrica de las extremidades y resistencia insulínica grave ${ }^{51}$. Este hallazgo, junto con la observación de una mayor frecuencia de mutaciones en genes asociados a lipodistrofia parcial en sujetos con síndrome metabólico grave pero sin fenotipo lipodistrófico clásico ${ }^{52}$, apoya la idea de que las lipodistrofias pueden ser confundidas con resistencia insulínica común asociada a obesidad central. Incluso, la diabetes mellitus tipo 2 "corriente" pudiera ser concebida como una forma de lipodistrofia en la que, pese a existir adiposidad corporal excesiva, existe incapacidad para remodelar fisiológicamente el tejido adiposo, determinando resistencia insulínica ${ }^{53}$.

\section{Conclusiones}

1. Las lipodistrofias primarias son enfermedades infrecuentes pero que determinan complicaciones estéticas, metabólicas, cardiacas y renales graves.

2. La resistencia insulínica en lipodistrofia determina diabetes mellitus, dislipidemia, esteatosis hepática con o sin hepatitis y ovario poliquístico. Estas complicaciones pueden ser refractarias al tratamiento médico convencional.

3. Pacientes con lipodistrofia parcial pueden ser confundidos con síndrome metabólico o diabetes mellitus tipo 2 común. Por lo tanto, en pacientes con alteraciones metabólicas comunes (resistencia insulínica, diabetes mellitus, hipertrigliceridemia-dislipidemia mixta, síndrome de ovario poliquístico) pero en contextos clínicos inusuales (bajo o normo peso, jóvenes, historias familiares sugerentes de herencia autosómica dominante o alteraciones metabólicas de gravedad desproporcionada para el contexto clínico del paciente), debe considerarse la posibilidad de un síndrome lipodistrófico.

Agradecimientos: Los autores agradecen a la Dra. Verónica Meriq por su valiosa colaboración clínica y científica en los casos chilenos de lipodistrofia.

\section{Referencias}

1. Cortés VA, Curtis DE, Sukumaran S, Shao X, Parameswara V, Rashid S, et al. Molecular mechanisms of hepatic steatosis and insulin resistance in the AGPAT2-deficient mouse model of congenital generalized lipodystrophy. Cell Metab 2009; 9: 165-76. 
2. Cortés VA, Cautivo KM, Rong S, Garg A, Horton JD, Agarwal AK. Leptin ameliorates insulin resistance and hepatic steatosis in Agpat2-/- lipodystrophic mice independent of hepatocyte leptin receptors. J Lipid Res 2014; 55: 276-88.

3. Fernández-Galilea M, Tapia P, Cautivo K, Morselli E, Cortés VA. AGPAT2 deficiency impairs adipogenic differentiation in primary cultured preadipocytes in a non-autophagy or apoptosis dependent mechanism. Biochem Biophys Res Commun 2015; 467: 39-45.

4. Cautivo KM, Lizama CO, Tapia PJ, Agarwal AK, Garg A, Horton JD, et al. AGPAT2 is essential for postnatal development and maintenance of white and brown adipose tissue. Mol Metab 2016; 5: 491-505.

5. Unger RH, Scherer PE. Gluttony, sloth and the metabolic syndrome: a roadmap to lipotoxicity. Trends Endocrin Met 2010; 21: 345-52.

6. Richter T, Janoudi G, Amegatse W, Nester-Parr S. Characteristics of drugs for ultra-rare diseases versus drugs for other rare diseases in HTA submissions made to the CADTH CDR. Orphanet J Rare Dis 2018; 13: 15.

7. Garg A. Lipodystrophies: Genetic and Acquired Body Fat Disorders. J Clin Endocr Metab 2011; 96: 3313-25.

8. Meehan CA, Cochran E, Kassai A, Brown RJ, Gorden P. Metreleptin for injection to treat the complications of leptin deficiency in patients with congenital or acquired generalized lipodystrophy. Expert Rev Clin Phar 2016; 9: 59-68.

9. Lupsa BC, Sachdev V, Lungu AO, Rosing DR, Gorden P. Cardiomyopathy in congenital and acquired generalized lipodystrophy: a clinical assessment. Medicine 2010; 89: 245-50.

10. Musso C, Javor E, Cochran E, Balow JE, and Gorden P. Spectrum of renal diseases associated with extreme forms of insulin resistance. Clin J Am Soc Nephro 2006; 1: 616-22.

11. Berardinelli W. An undiagnosed endocrinometabolic syndrome: report of 2 cases. J Clin Endocr Metab 1954; 14: 193-204.

12. Seip M. Lipodystrophy and gigantism with associated endocrine manifestations. A new diencephalic syndrome? Acta Paediatrica 1959; 48: 555-74.

13. McNally M, Mannon RB, Javor ED, Swanson SJ, Hale DA, Gorden P, et al. Successful Renal Transplantation in a Patient with Congenital Generalized Lipodystrophy: A Case Report. Am J Transplant 2004; 4: 447-9.

14. Maguire M, Lungu A, Gorden P, Cochran E, Stratton P. Pregnancy in a woman with congenital generalized lipodystrophy: leptin's vital role in reproduction. Obstet Gynecol 2012; 119: 452-5.

15. Javor ED, Moran SA, Young JR, Cochran EK, DePaoli
AM, Oral EA, et al. Proteinuric nephropathy in acquired and congenital generalized lipodystrophy: baseline characteristics and course during recombinant leptin therapy. J Clin Endocrinol Metab 2004; 89: 3199-207.

16. Saito I, Saruta T, Iyori S, Sakaguchi H. The renal lesion associated with total lipodystrophy. J Am Geriatr Soc 1977; 25: 175-8.

17. Ishii J, Abe M, Kubo M, Sumimoto T, Hamada M, Hiwada $\mathrm{K}$, et al. Total lipodystrophy with heart failure and renal failure: report of a case. Jpn J Med 1989; 28: 534-7.

18. Lee HL, Waldman MA, Auh S, Balow JE, Cochran EK, Gorden $\mathrm{P}$, et al. Effects of metreleptin on proteinuria in patients with lipodystrophy. J Clin Endocrinol Metab 2019.

19. Coresh J, Heerspink HJL, Sang Y, Matsushita K, Arnlov J, Astor BC, et al. Change in albuminuria and subsequent risk of end-stage kidney disease: an individual participant-level consortium meta-analysis of observational studies. Lancet Diabetes Endo 2019; 7: 115-27.

20. Khalife WI, Mourtada MC, Khalil J. Dilated cardiomyopathy and myocardial infarction secondary to congenital generalized lipodystrophy. Tex Heart I J 2008; 35: 196-9.

21. Weir-Mitchell S. Singular case of absence of adipose matter in upper half of the body. Am J Med Sci 1885; 90: 105-6.

22. Subramanyam L, Simha V, Garg A. Overlapping syndrome with familial partial lipodystrophy, Dunnigan variety and cardiomyopathy due to amino-terminal heterozygous missense lamin $\mathrm{A} / \mathrm{C}$ mutations. Clin Genet 2010; 78: 66-73.

23. Garg A. Gender differences in the prevalence of metabolic complications in familial partial lipodystrophy (Dunnigan variety). J Clin Endocrinol Metab 2000; 85: 1776-82.

24. Kobberling J, Willms B, Kattermann R, Creutzfeldt W. Lipodystrophy of the extremities. A dominantly inherited syndrome associated with lipatrophic diabetes. Humangenetik 1975; 29: 111-20.

25. Herbst KL, Tannock LR, Deeb SS, Purnell JQ, Brunzell JD, Chait A. Kobberling type of familial partial lipodystrophy: an underrecognized syndrome. Diabetes Care 2003; 26: 1819-24.

26. Dunnigan MG, Cochrane MA, Kelly A, Scott JW. Familial lipoatrophic diabetes with dominant transmission. A new syndrome. Q J Med 1974; 43: 33-48.

27. Garg A. Lipodystrophies. Am J Med 2000; 108: 143-52.

28. Garg A, Peshock RM, Fleckenstein JL. Adipose tissue distribution pattern in patients with familial partial lipodystrophy (Dunnigan variety). J Clin Endocrinol Metab 1999; 84: 170-4.

29. Ursich MJ, Fukui RT, Galvao MS, Marcondes JA, Santo- 
mauro AT, Silva ME, et al. Insulin resistance in limb and trunk partial lipodystrophy (type 2 Kobberling-Dunnigan syndrome). Metab Clin Exp 1997; 46: 159-63.

30. Peters JM, Barnes R, Bennett L, Gitomer WM, Bowcock AM, Garg A. Localization of the gene for familial partial lipodystrophy (Dunnigan variety) to chromosome 1q21-22. Nat Genet 1998; 18: 292-5.

31. Cao H, Hegele RA. Nuclear lamin A/C R482Q mutation in canadian kindreds with Dunnigan-type familial partial lipodystrophy. Hum Mol Gen 2000; 9: 109-12.

32. Agarwal AK, Garg A. A novel heterozygous mutation in peroxisome proliferator-activated receptor-gamma gene in a patient with familial partial lipodystrophy. J Clin Endocrinol Metab 2002; 87: 408-11.

33. Gandotra S, Le Dour C, Bottomley W, Cervera P, Giral P, Reznik Y, et al. Perilipin deficiency and autosomal dominant partial lipodystrophy. N Engl J Med 2011; 364: 740-8.

34. Rubio-Cabezas O, Puri V, Murano I, Saudek V, Semple RK, Dash S, et al. Partial lipodystrophy and insulin resistant diabetes in a patient with a homozygous nonsense mutation in CIDEC. EMBO Mol Med 2009; 1: 280-7.

35. Albert JS, Yerges-Armstrong LM, Horenstein RB, Pollin TI, Sreenivasan UT, Chai S, et al. Null Mutation in Hormone-Sensitive Lipase Gene and Risk of Type 2 Diabetes. N Engl J Med 2014; 370: 2307-15.

36. Cao H, Alston L, Ruschman J, Hegele RA. Heterozygous CAV1 frameshift mutations (MIM 601047) in patients with atypical partial lipodystrophy and hypertriglyceridemia. Lipids Health Dis 2008; 7: 3.

37. Misra A, Garg A. Clinical features and metabolic derangements in acquired generalized lipodystrophy: case reports and review of the literature. Medicine 2003; 82: 129-46.

38. Misra A, Peethambaram A, Garg A. Clinical features and metabolic and autoimmune derangements in acquired partial lipodystrophy: report of 35 cases and review of the literature. Medicine 2004; 83: 18-34.

39. Savage DB, Semple RK, Clatworthy MR, Lyons PA, Morgan BP, Cochran EK, et al. Complement Abnormalities in Acquired Lipodystrophy Revisited. J Clin Endocrinol Metab 2009; 94: 10-6.

40. Lenane P, Murphy GM. Partial lipodystrophy and renal disease. Clin Exp Dermatol 2008; 25: 605-7.

41. Oliveira J, Freitas P, Lau E, Carvalho D. Barraquer-Simons syndrome: a rare form of acquired lipodystrophy. BMC Res Notes 2016; 9: 175.
42. Visser ME, Kropman E, Kranendonk ME, Koppen A, Hamers N, Stroes ES, et al. Characterisation of non-obese diabetic patients with marked insulin resistance identifies a novel familial partial lipodystrophy-associated PPAR gamma mutation (Y151C). Diabetologia 2011; 54: 1639-44.

43. Dutour A, Roll P, Gaborit B, Courrier S, Alessi MC, Tregouet DA, et al. High prevalence of laminopathies among patients with metabolic syndrome. Hum Mol Gen 2011; 20: 3779-86.

44. Cortés VA, Smalley SV, Goldenberg D, Lagos CF, Hodgson MI, Santos JL. Divergent Metabolic Phenotype between Two Sisters with Congenital Generalized Lipodystrophy Due to Double AGPAT2 Homozygous Mutations. A Clinical, Genetic and In Silico Study. PloS one 2014; 9: e87173.

45. Goldenberg D, Santos JL, Hodgson MI, Cortés VA. [Novel physiological and therapeutic implications of leptin]. Rev Med Chile 2014; 142: 738-47.

46. Utzschneider KM, Trence DL. Effectiveness of gastric bypass surgery in a patient with familial partial lipodystrophy. Diabetes Care 2006; 29: 1380-2.

47. Ciudin A, Baena-Fustegueras JA, Fort JM, Encabo G, Mesa J, Lecube A. Successful treatment for the Dunnigan-type familial partial lipodystrophy with Roux-en-Y gastric bypass. Clin Endocrinol (Oxf) 2011; 75: 403-4.

48. Melvin A, Adams C, Flanagan C, Gaff L, Gratton B, Gribble F, et al. Roux-en-Y Gastric Bypass Surgery in the Management of Familial Partial Lipodystrophy Type 1. J Clin Endocrinol Metab 2017; 102: 3616-20.

49. Oral EA, Simha V, Ruiz E, Andewelt A, Premkumar A, Snell $\mathrm{P}$, et al. Leptin-replacement therapy for lipodystrophy. N Engl J Med 2002; 346: 570-8.

50. Takeyari S, Takakuwa S, Miyata K, Yamamoto K, Nakayama H, Ohata Y, et al. Metreleptin treatment for congenital generalized lipodystrophy type 4 (CGL4): a case report. Clin Pediatr Endocrinol 2019; 28: 1-7.

51. Strickland LR, Guo F, Lok K, Garvey WT. Type 2 diabetes with partial lipodystrophy of the limbs: a new lipodystrophy phenotype. Diabetes Care 2013; 36: 2247-53.

52. Decaudain A, Vantyghem MC, Guerci B, Hecart AC, Auclair M, Reznik Y, et al. New metabolic phenotypes in laminopathies: LMNA mutations in patients with severe metabolic syndrome. J Clin Endocrinol Metab 2007; 92: 4835-44.

53. Danforth E Jr. Failure of adipocyte differentiation causes type II diabetes mellitus? Nat Genet 2000; 26: 13. 